\title{
Left ventricular pseudoaneurysm complicating infective pericarditis
}

\author{
A do Nascimento Moraes, A Gomes Ferreira Jr, S M A Gomes Ferreira
}

\begin{abstract}
Cross sectional echocardiography demonstrated a pseudoaneurysm of the left ventricular posterolateral wall close to the atrioventricular junction in a 4 year old girl with infective pericarditis complicating lobar pneumonia. Colour flow Doppler demonstrated bidirectional flow across the communication hole. Surgical resection was successful.

(Heart 1999;82:393-394)
\end{abstract}

Fundação Luiz Décourt, Serviço de

Cardiologia

A do Nascimento Moraes

A Gomes Ferreira Jr

Faculdade de Medicina da Universidade

Federal do Pará

S M A Gomes Ferreira

Correspondence to:

Dr S M A Gomes Ferreira, Rua Jerônimo Pimentel, 519 , Umarizal, Belém, Pará,

Brazil-CEP: 66055-202.

Accepted for publication 19 March 1999

Keywords: pseudoaneurysm; pericarditis; echocardiography

A ventricular pseudoaneurysm can develop after myocardial rupture, when a combination of pericardial adherence and thrombus formation prevents fatal tamponade but allows progressive expansion. ${ }^{1}$ Similarly a pseudoaneurysm can develop after surgical resection of right ventricular outflow tract obstruction in tetralogy of Fallot or other complex cyanotic heart defects or after closed pulmonary valvotomy. ${ }^{2}$ Pseudoaneurysm formation as a complication of infective endocarditis has been reported, ${ }^{3}$ but to our knowledge a left ventricu-

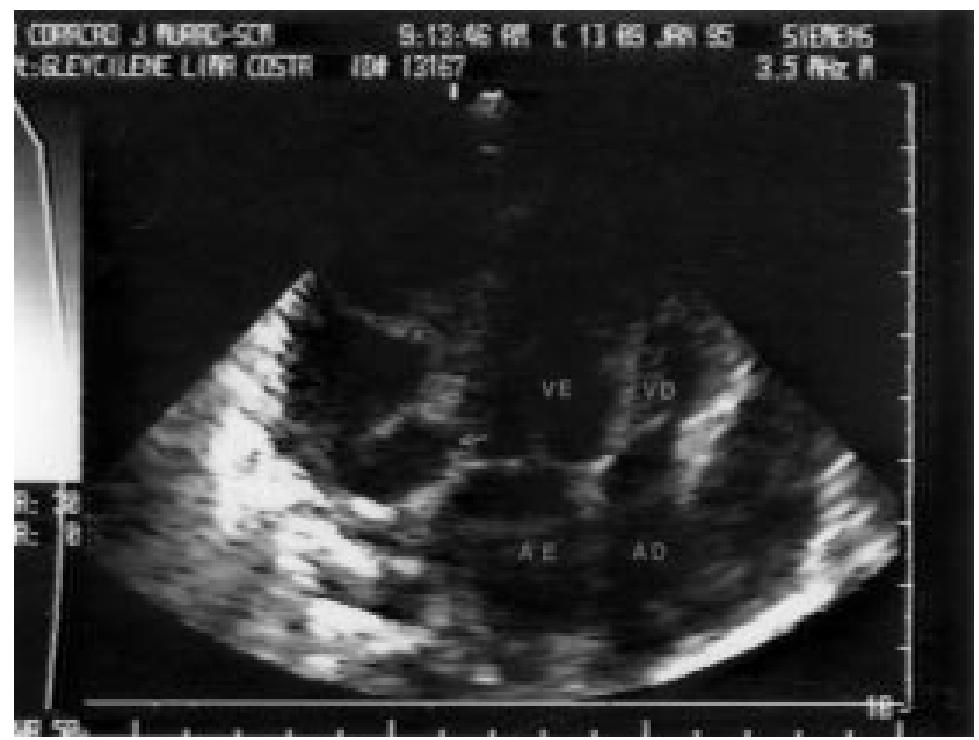

Figure 1 Cross sectional echocardiography (apical four chamber view) showing large pericardial effusion and the communication between the left ventricle and the pseudoaneurysm (arrow). VE, left ventricle; VD, right ventricle; $A E$, left atrium; $A D$, right atrium. lar pseudoaneurysm developing in a child with an otherwise normal heart has not been reported. We describe a case of pseudoaneurysm of the left atrioventricular junction that developed as a complication of infective pericarditis.

\section{Case report}

The patient was a previously well 4 year old girl who presented with right lower lobe pneumonia. She was treated with ampicillin and gentamicin, but after two weeks she became increasingly breathless with hepatomegaly and raised jugular venous pressure. She also developed a septic arthritis of her left elbow. Plain chest radiography showed considerable enlargement of the cardiac shadow, and cross sectional echocardiography showed a large pericardial effusion (fig 1). Intracardiac anatomy was otherwise normal, there were no vegetations but Doppler signals showed restrictive ventricular filling.

The septic arthritis and pericardial effusion were drained and Staphylococcus aureus was cultured. The antibiotic was changed to flucloxacillin but after three weeks the patient continued to have fever and signs of congestive heart failure (raised jugular venous pressure and hepatomegaly). Repeat cross sectional echocardiography showed a large pericardial effusion with some septation or fibrous strands. A narrowed discontinuity was seen in the posterolateral wall of the basal part of the left ventricle close to the atrioventricular junction. Associated with this was a localised bulging of the pericardium with a narrow communication from the ventricle in to the pericardial space consistent with the diagnosis of a false aneurysm of the left ventricle. Colour flow mapping showed bidirectional flow through the communication (fig 2). Left ventriculography confirmed the diagnosis (fig 3 ).

The patient underwent resection of the portion of the pericardium that formed the wall of the false aneurysm, the purulent pericardial effusion was drained, and the communication between left ventricular posterolateral wall was closed with autologous pericardium. Cardiopulmonary bypass was not needed.

Microscopic examination of the resected specimen showed this to be fibrous tissue, representing adhesion of the parietal and visceral pericardial layers of the pericardium, which 


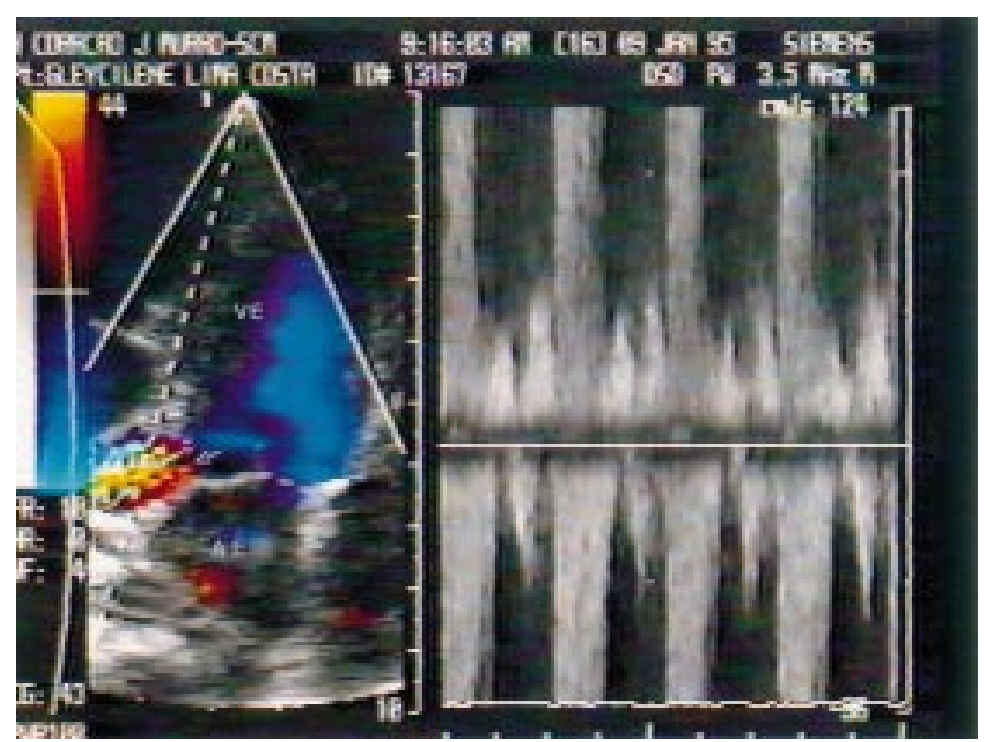

Figure 2 Colour flow mapping and pulsed Doppler recording at the origin of the pseudoaneurysm (arrow). VE, left ventricle; $A E$, left atrium.

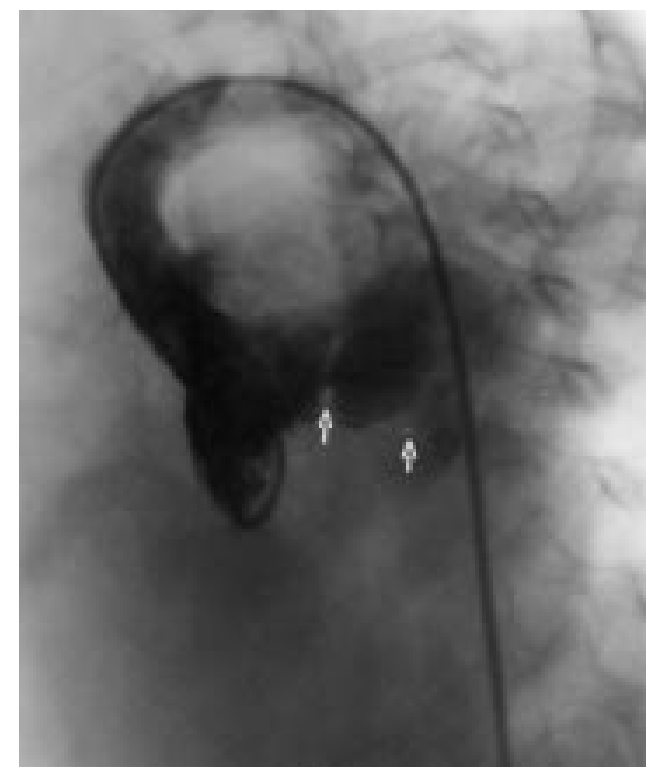

Figure 3 Left ventriculogram in the left anterior oblique view showing a large aneurysm of the posterolateral wall (arrows).

obliterated the percardial sac; this had prevented complete rupture. The postoperative course was uneventful. Two weeks after surgery, cross sectional echocardiography showed normal intracardiac anatomy, no vegetations, no pericardial effusion and normal ventricular function. Six months later on outpatient review the findings were similar.

\section{Discussion}

A pseudoaneurysm of the ventricle is formed when there is rupture of the myocardial wall with the discontinuity being roofed over by pericardium and mural thrombus or fibrous tissue without myocardial elements. False aneurysms adjacent to the atrioventricular junction have been described after mitral valve replacement ${ }^{4}$ and myocardial infarction necessitating mitral valve replacement. ${ }^{5}$ False aneurysm complicating infective endocarditis has almost always proved fatal because of the propensity to rupture. ${ }^{36}$ We are not aware of previous reports of pseudoaneurysm as a complication of infective pericarditis, although such a finding may explain some deaths with this condition.

Staphylococcus aureus is the most common organism causing infective pericarditis. Because of its virulence and ability to destroy or disrupt cardiac structures, complications may occur rapidly after infection. In the present case the primary infection was thought to be pulmonary. Early diagnosis was made because of the rapid development of signs compatible with congestive heart failure, which prompted echocardiography and colour Doppler, which allowed distinction between a true and false aneurysm.

Pseudoaneurysms related to an infective process carry a bad prognosis, but our case showed that early surgical intervention can change this course.

We are very grateful to Dr Elliot Shinebourne for his careful review of the manuscript.

1 De Vries AG, Saelman JPM, Southerland GR. The value of colour flow mapping in the diagnosis of a combined pseudoaneurysm and large true left ventricular aneurysm. Eur Heart f 1991;12:280-2.

2 Sadiq M, Fenton AC, Firmin RK. False aneurysm of the right ventricle outflow tract after total correction of tetralogy of Fallot: diagnosis by echocardiography and succsseful repair by neck cannulation of cardiopulmonary bypass. $\mathrm{Br}$ repair by neck cannulation

3 Qizilbash AH, Schwartz CJ. False aneurysm of left ventricle due to perforation of mitral-aortic intervalvular fibrosa with rupture and cardiac tamponade. Rare complication of infective endocarditis. Am f Cardiol 1973;32:110-13.

4 Sakai K, Nakamura K, Ishizuka N, et al. Echocardiographic findings and clinical features of left ventricular pseudoaneurysm after mitral valve replacement. Am Heart $f$ 1992;124:975-82.

5 Esakof DD, Vannan MA, Pandian NG, et al. Visualization of left ventricle pseudoaneurysm with panoramic transesophageal echocardiography. $\mathcal{F}$ Am Soc Echocardiogr. 1994; 7:174-8.

6 Mundith ED, Mayer SH, Hopkins FT. Surgical management of post-infarction leaking left ventricular false aneurysm. F Cardiovasc Surg 1989;30:796-7. 